\title{
EFECTO DEL HERBICIDA GLIFOSATO EN HONGOS ENDÓFITOS DE RAÍZ Y KEIKIS DE EPIDENDRUM MELINANTHUM (ORCHIDACEAE)
}

\author{
Erika Perea-Morera ${ }^{1,2,5} \&$ Joel Tupac Otero ${ }^{2,3,4}$ \\ ${ }^{1}$ Facultad de Ingeniería y administración, Universidad Nacional de Colombia sede Palmira. \\ Carrera 32 No 12-00 Chapinero, Vía Candelaria. Palmira, Valle del Cauca, Colombia \\ ${ }^{2}$ Grupo de Investigación en Orquídeas, Ecología y Sistemática Vegetal; Departamento de Ciencias \\ Biológicas, Universidad Nacional de Colombia Sede Palmira Calle 32 N 12-00, \\ Palmira-Valle del Cauca-Colombia \\ ${ }^{3}$ Departamento de Ciencias Biologicas, Universidad Nacional de Colombia sede Palmira. \\ Carrera 32 No 12-00 Chapinero, Vía Candelaria. Palmira, Valle del Cauca, Colombia \\ ${ }^{4}$ Instituto de Ciencias Ambientales IDEA, Universidad Nacional de Colombia Sede Palmira \\ ${ }^{5}$ Author for correspondence: epeream@unal.edu.co
}

RESUMEN. El uso de herbicidas ha tenido un impacto grande sobre la producción agrícola y sin duda ha aumentado la producción en muchas cosechas. Desafortunadamente, la aplicación de herbicidas puede tener efectos negativos sobre otras especies nativas aledañas al cultivo. Con el fin de establecer si el herbicida glifosato afecta negativamente a la orquídea terrestre Epidendrum melinanthum y sus hongos endófitos aislados de la raíz, se evaluaron tres dosis de Round Up® en keikis y en hongos endófitos previamente aislados. Las dosis de aplicación se determinaron a partir de la utilizada para la maduración en caña de azúcar (1.5 1/Ha equivalentes a $544.5 \mathrm{~g}$ de glifosato/1) de la siguiente manera: a) control (sin herbicida), b) dosis de aplicación en caña de un décimo y c) una centésima parte de la dosis de maduración. Se observó en keikis el cambio de coloración en tallo y en hojas, adicional a la caída de las mismas. El Round Up generó afectación en uno de los tres hongos estudiados en forma de un halo de inhibición. Se concluye, que el glifosato afecta negativamente las estructuras foliares de los keikis de manera rápida al aplicar el herbicida sobre las hojas, en adición a la inhibición de hongos endófitos de Epidendrum melinanthum. Estos aspectos sugieren el potencial negativo del herbicida sobre la especie estudiada y su microflora asociada.

Palabras claves: efectos del glifosato, Epidendrum melinanthum, inhibición de hongos, keikis, orquídeas, Round Up

Introducción. Los herbicidas son económicamente rentables para la erradicación de algunas malezas en cultivos productivos, especialmente en cultivos que son de lento crecimiento, pues en estos cultivos se favorece la aparición de hierbas que pueden llegar a reducir el rendimiento en un 60-70\% (Hajebi et al. 2015). Los herbicidas pueden clasificarse de múltiples maneras, bien sea por la utilización, por las propiedades químicas y el modo de acción (Labrada et al. 1996) y pueden afectar a las plantas dependiendo de las características que cada especie tenga, la edad, la cantidad, las condiciones ambientales, y el tiempo transcurrido después de la aplicación (FAO 2016). Por lo tanto, existen especies que son resistentes a algunos herbicidas como es el caso de Zea mays L. y
Glycine $\max \mathrm{L}$. que presentan resistencia al glifosato (Obour et al. 2016). Si bien los herbicidas juegan un importante papel en el control de malezas (Freitas et al. 2016) y como es el caso del glifosato, puede actuar como fuente de carbono, estimular la actividad microbiológica del suelo y la respiración microbiana (Obour et al. 2016). Pero el uso de herbicidas puede impactar negativamente los ecosistemas, acumularse en la cadena alimentaria y afectar gravemente la salud humana (Freitas et al. 2016).

Las especies de la familia Orchidaceae tienen grandes amenazas dado que son extremadamente susceptibles a cambios en su entorno en comparación con otras plantas, las afectan principalmente las malezas, agentes patógenos, cambio climático, 
incendios, animales, perdida de hábitat, prácticas agrícolas, el uso de fertilizantes y el turismo (Pillon et al. 2006, Ranking et al. 2015), por lo cual, tienen una mayor proporción de géneros y especies amenazadas que la mayoría de familias (Zhang et al. 2015). Dada la alta vulnerabilidad y la limitada distribución de la mayoría de las especies, efectos locales pueden resultar en extinciones de especies (Zhang et al. 2015). Los efectos negativos sobre las poblaciones de orquídeas en general se refieren a la deforestación o la sobreexplotación, pero hay efectos indirectos que podría estar causando la reducción en tamaño poblacional que son más sutiles. El uso de herbicidas en grandes extensiones como la producción de caña de azúcar podría tener un impacto en la flora que rodea estas áreas agrícolas, por lo tanto, es imperativo realizar un análisis de los impactos negativos generados por prácticas agrícolas, esto con el fin de diseñar políticas de conservación.

El Valle del Río Cauca se caracteriza por ser la zona donde se encuentra ubicado el principal sector azucarero de Colombia, el cual cuenta con aproximadamente 225,560 Ha de tierra sembradas en caña (ASOCAÑA 2012), dentro del proceso de cultivo de la caña de azúcar se encuentra la maduración, proceso fisiológico en el cual se favorece la concentración de sacarosa en la caña (Cassalett et al. 1995). Desde 1920 se ha venido investigando sobre el uso de madurantes, siendo el glifosato el que presenta efectos más consistentes y de mayor efectividad para el cultivo de caña, pues aumenta el rendimiento de sacarosa en el cultivo, logrando el aumento de hasta cinco veces más de sacarosa en el tallo (Su et al. 1992, Cassalett et al. 1995), con dosis entre 1.0 y $2.01 / \mathrm{Ha}$ con aplicaciones entre la sexta y catorceava semana (Cassalett et al. 1995).

Los productos comerciales implementados para la maduración son el Round Up o glifosol, cuyo objetivo es concentrar en el tallo un mayor contenido de azúcar en la caña (Cassalett et al. 1995). El componente activo de los productos utilizados para la maduración es el glifosato ( $N-$ (fosfonometil) glicina), este es mayormente utilizado como un herbicida general y ha sido utilizado desde 1974 en numerosas presentaciones comerciales y distribuido a nivel mundial (Steinmann et al. 2012). La acción del glifosato se desencadena por la inhibición de la enzima 5-enolpiruvilshikimato- 3-fosfato sintetasa (EPSPS), mediante la ruta del ácido shikímico que impide la síntesis de tres aminoácidos esenciales (fenilalanina, tirosina y triptófano), lo cual impide crecimiento e inhibe la capacidad de eliminar toxinas (Cox 1998). El glifosato en dosis de $1 \mathrm{~kg} / \mathrm{Ha}$ es un herbicida no selectivo, además, es absorbido por las hojas de la planta y se transloca a las raíces, lo cual permite una actividad sistémica (Steinmann et al. 2012).

Beyrle et al. (1994) demostraron que la incidencia del glifosato inhibe la germinación de semillas de la orquídea terrestre de la zona templada Orchis morio, además, evalúan el efecto del glifosato sobre el crecimiento de algunos hongos micorrízicos y la inhibición de la iniciación micorrízica. Los objetivos de este estudio son: evaluar el efecto del glifosato sobre Epidendrum melinanthum, respecto al número, color y curvatura de hojas, así como la producción de keikis. Adicionalmente, se pretende determinar si hay un efecto del glifosato en el crecimiento de hongos endófitos.

\section{Materiales y métodos}

Epidendrum melinanthum Schltr. (Orchidaceae: Epidendroideae: Laeliinae) (Romero \& Ferreira 2006) es una orquídea terrestre presente en bosque seco tropical que presenta óptimo crecimiento en franjas comprendidas entre 1100 y $1300 \mathrm{msnm}$, con inflorescencia racemosa, labelos divididos en tres lóbulos y color amarillo en sépalos y pétalos (Fig. 1) (Reina-Rodríguez \& Otero 2011).

El estudio se realizó con E. melinanthum como especie modelo, dado que es una especie ampliamente distribuida en el Valle del Cauca, además de ser una especie que es vulnerable a las actividades agrícolas de la caña pues su hábitat está en zonas aledañas al monocultivo.

Material vegetal. Colección de raíces. Las raíces se colectaron en el corregimiento de Los Ceibos ubicado en el municipio de Palmira-Valle del Cauca el mes de noviembre del 2014. Las raíces se obtuvieron de plantas que se encontraron en buen estado y sin deteriorar su sistema radicular (Fig. 2). Las muestras se conservaron en bolsas de polietileno etiquetadas con el fin de mantener la humedad hasta ser procesadas en el laboratorio el mismo día (Mosquera et al. 2010, Han et al. 2016). 


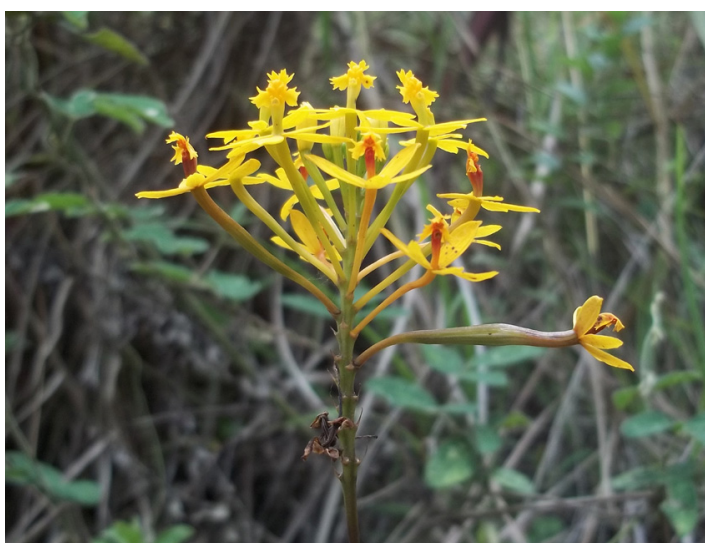

Figura 1. Flor de Epidendrum melinanthum en el Valle del Cauca.

Colección de keikis. Los keikis de E. melinanthum de alrededor de $10 \mathrm{~cm}$, se obtuvieron de la población natural de La Vorágine-Cali y Tablones Palmira en noviembre del 2014. El material vegetal se depositó en bolsas de papel, se etiquetaron y se transportaron en una nevera de icopor hasta el laboratorio.

Aislamiento de hongos. Dos morfotipos de hongos endófitos fueron aislados de raíces de E. melinanthum y uno de papa. Cada raíz se cortó en secciones de 1 $\mathrm{cm}$ y se etiquetaron, se procedió a sembrar en agar de dextrosa de patata (PDA) dentro de la cámara de flujo laminar (Valadares et al. 2015), con antelación las raíces se esterilizaron superficialmente en etanol al $70 \%$ por $1 \mathrm{~min}$, hipoclorito de sodio al $2.5 \%$ por $2 \mathrm{~min}$, etanol al $70 \%$ por 1 min y se realizaron tres lavados con agua destilada estéril (Otero et al. 2002, Otero \& Bayman 2009, Tan et al. 2014). Al azar se colocaron trozos de cada sección en PDA, se incubaron a $25^{\circ} \mathrm{C}$ y se observaron diariamente. Cada nueva hifa se transfirió a PDA para obtener los hongos purificados y su posterior identificación (Otero \& Bayman 2009, Tan et al. 2014).

Caracterización morfológica de los hongos endófitos aislados. La caracterización morfológica de los tres hongos aislados se llevo a cabo mediante la observación de placas en el microscopio. Los hongos de Rhizoctonia presentan anamórfismos y telemórfismos, pero se caracterizan por tener un ángulo de ramificación recto, presentan hifas con constricción en tabiques, en ángulo recto ramificado de las hifas después de los tabiques

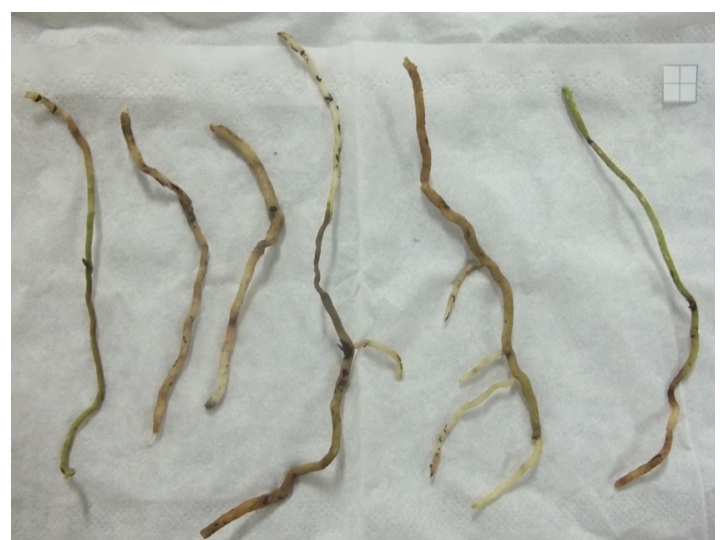

FIGURA 2. Raíces de Epidendrum melinanthum.

y con frecuencia presentan hifas inflamadas bien conocidas como monillioid (Otero et al. 2002, Otero et al. 2004, Otero et al. 2005, Otero et al. 2007, Pereira et al. 2014).

Concentración de glifosato. Efecto del glifosato en el crecimiento de hongos endófitos. El efecto del glifosato sobre el crecimiento de hongos endófitos aislados se realizó de dos maneras, la primera consistió en poner un disco de papel filtro estéril con las dosis estudiadas de glifosato en cajas Petri que contenían PDA y se procedió a sembrar los hongos; se realizaron observaciones de la formación del halo de inhibición en las tres dosis y el control. La segunda prueba, consistió en disolver el glifosato en el PDA justo cuando se estuviera sirviendo el medio, esto con el fin de observar el diámetro de crecimiento de los tres morfotipos de hongos aislados, uno de papa y dos de E. melinanthum.

Efecto del glifosato sobre el número, color y curvatura de hojas y producción de keikis de $E$. melinanthum. Las plántulas se trasplantaron en macetas que contenían sustrato para orquídeas de contextura media adquirida en el orquideograma de Cali. Posteriormente, las macetas se aclimataron en el invernadero de la Universidad Nacional de Colombia Sede Palmira con humedad relativa de $70-80 \%$, aproximadamente de $12 \mathrm{~h} \mathrm{~d}^{-1}$ fotoperíodo, 120-180 mol m-2 $\mathrm{s}^{-1}$ intensidad de luz PPF (luz solar sombreada) y régimen de temperatura $28 \pm 2$ ${ }^{\circ} \mathrm{C}$ durante el día y $25 \pm 2{ }^{\circ} \mathrm{C}$ durante la noche (Panwar et al. 2012). Posteriormente, los keikis se 
organizaron de manera homogénea y se dividieron con láminas de vidrios para que las dosis de glifosato aplicadas no se mezclaran.

Se realizó la aplicación de Round Up donde la dosis 1 , es equivalente a $1.5 \mathrm{1} / \mathrm{Ha}(544.5 \mathrm{~g} / \mathrm{l})$, la dosis típicamente usada en la cosecha de caña de azúcar para la maduración, entonces el diseño incluye tres dosis experimentales 1, $1 / 10,1 / 100$ y el control con $\mathrm{H}_{2} \mathrm{O}$ destilada estéril con 30 repeticiones cada una, se realizaron dos aplicaciones con una diferencia temporal de 15 días y se realizó seguimiento semanal al número de hojas, color de las hojas y tallos, curvatura de hojas y producción de keikis, durante seis semanas posterior a la primera aplicación.

\section{Análisis histológico}

Al final del experimento se realizaron cortes histológicos con el fin de evidenciar las diferencias en tallo y hojas, respecto al control. El análisis se realizó a través de cortes histológicos de aproximadamente 100 $\mu \mathrm{m}$ que fueron posteriormente teñidos con azul de toluidina y observados en el microscopio (Puerto et al. 2001).

\section{Métodos estadísticos}

Se realizó el análisis de varianza (ANOVA) con un nivel de significación de $a=0.05$. Además, se realizó una comparación de medias con la prueba de rango múltiple de Duncan para el análisis de la perdida de hojas en E. melinanthum y crecimiento de hongos endófitos, y por otra parte, se realizó un análisis de correspondencia múltiple cualitativa en el efecto de color, curvatura y producción de keikis; se efectuó mediante el paquete estadístico SAS (2000).

La prueba de rango múltiple de Duncan se realizó porque permite comparar todos los tratamientos entre sí, además, el número que se analizó no era profuso, por lo cual era ideal para obtener resultados que determinaran si existen diferencias significativas en la investigación. Paralelamente, se realizó la prueba de correspondencia múltiple cualitativa con el fin de observar en un mapa las variables estudiadas para identificar las asociaciones entre las mismas. Por consiguiente, la hipótesis nula $\left(\mathrm{H}_{0}\right)$ es: si el glifosato afecta negativamente el crecimiento de hongos endófitos de E. melinanthum, y en segundo lugar, la $\mathrm{H}_{0}$ para la prueba de pérdida de hojas equivale a; si el glifosato favorece la perdida de hojas de $E$. melinanthum.

\section{Resultados}

Caracterización morfológica de los hongos endófitos aislados

Se obtuvieron tres hongos aislados determinados como Rhizoctonia sp., de los cuales, dos se obtuvieron de las raíces de E. melinanthum, y uno de papa. Todos los hongos aislados cumplían las características descritas en la metodología.

Efecto del glifosato en el crecimiento de hongos endófitos

Se obtuvo que el glifosato a la concentración $544.5 \mathrm{~g} / \mathrm{l}$ inhibe significativamente el crecimiento del morfotipo (B) aislado de E. melinanthum con respecto a los hongos restantes, pues la probabilidad mayor que el F calculado $(\operatorname{Pr}>\mathrm{F})=0.0422<0.05$. El crecimiento en este morfotipo era más lento con respecto al control y forma un halo de inhibición a concentración 544.5 g/l (Fig. 3). Por otra parte, se observó que no hay diferencias significativas entre el control y las tres dosis de glifosato aplicadas, pues $\operatorname{Pr}>\mathrm{F}=0.9673>$ 0.05 , sin embargo, el $50 \%$ de los hongos, equivalente a la diferencia de los cuartiles tres y uno, crece con un diámetro menor en la concentración $544.5 \mathrm{~g} / 1$ con respecto al control, evidenciando que existen diferencias significativas entre las dos concentraciones pues las áreas de las cajas y sus respectivos bigotes no se interceptan (Fig. 4), a la concentración 544.4 $\mathrm{g} / \mathrm{l}$ de glifosato pueden llegar a afectar el crecimiento de los tres hongos aislados, como se observa en la Fig. 4, donde se muestran los diámetros medios de crecimiento de los hongos aislados.

Efecto delglifosato sobreelcolor, curvatura, producción de keikis y número de hojas de E. melinanthum

Se observaron daños en tallos y hojas de diversas sintomatologías, que a continuación se describen:

El color de tallo y hojas: Se presentaron cambios de color en hojas en tonalidades de amarillo con manchas violetas (Fig. 5A), puntos y manchas violetas (Fig. 5B), puntos y manchas blancas (Fig. $5 \mathrm{C}$ y $5 \mathrm{D}$ ), puntos negros (Fig. 5C), manchas con tonalidades café (Fig. 5C), en la zona de transición ente la hoja y el tallos se observa una tonalidad negra en los tejidos (Fig. 5D), por su parte, en los tallo se evidenció la aparición de manchas negras (Fig. 5D). 

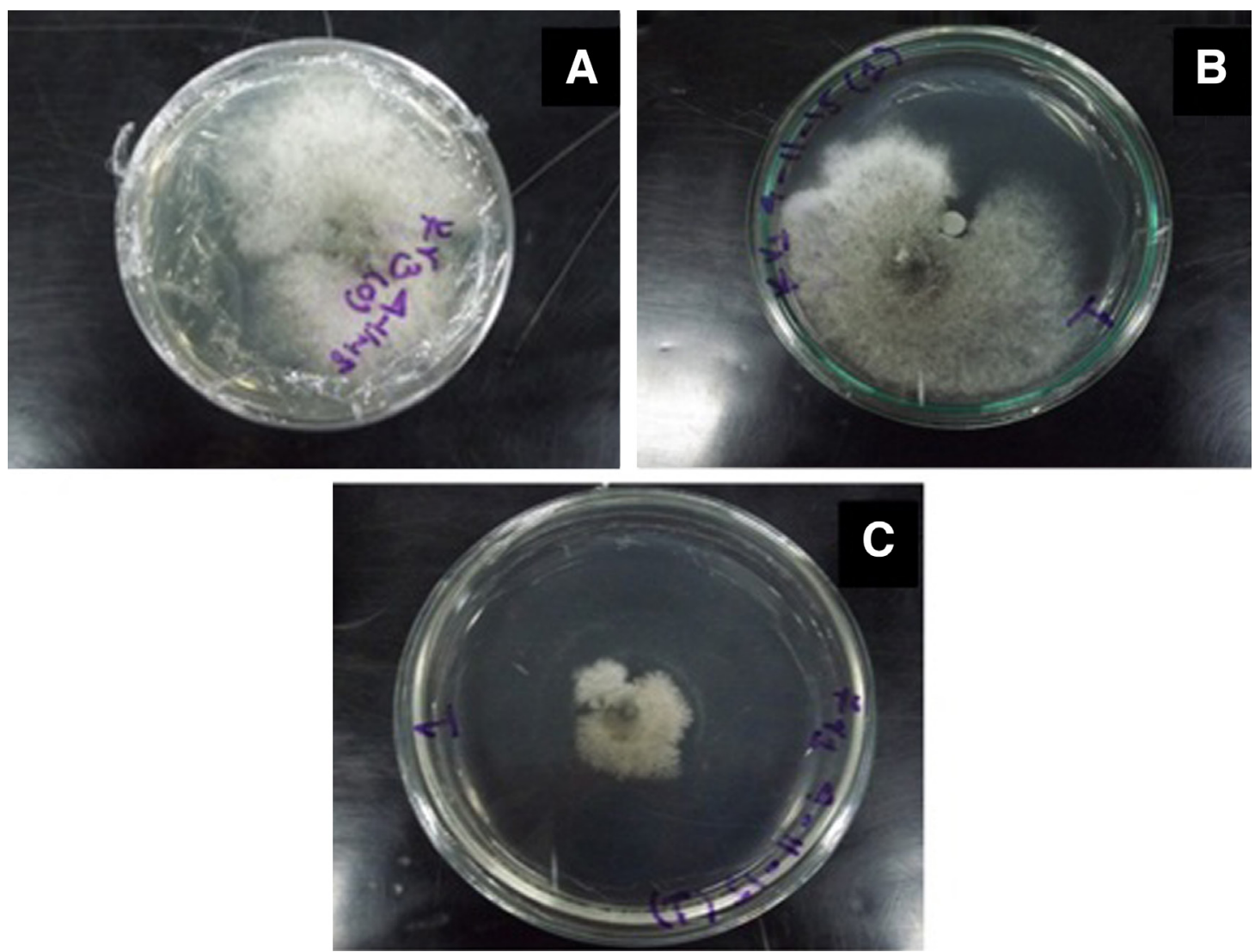

FiguRA 3. A. Hongo sembrado en PDA (control). B. Halo de inhibición por dosis 1 de glifosato. C. Disminución de crecimiento con dosis 1 .

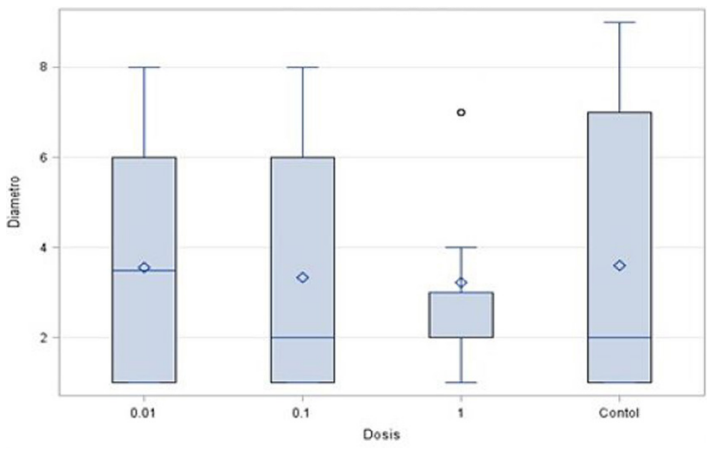

FIgURA 4. Distribución de diámetros promedio acuerdo a las dosis aplicadas. La línea del centro de la caja representa la mediana, el diamante representa la media y los bigotes representan los percentiles 5\% - $25 \%$ y $75 \%$ - $95 \%$ y la caja representa el $50 \%$ equivalente a la resta del cuartil tres y cuartil uno. Los puntos que se encuentran por fuera de los bigotes son datos atípicos.
Curvatura de hojas: luego de la aplicación del glifosato se observo que las hojas se tornaron curveadas y cóncavas con diversas tonalidades (Fig. 5B).

Producción de keikis: se encontró que la producción de keikis era mayor en dosis $1 / 10$, seguido de la concentración de $544.5 \mathrm{~g} / 1$ equivalente a la dosis 1 comparados con el control (Fig. 6), sin embargo, la producción de keikis no es representativa. También, se presentan semejanzas entre los efectos obtenidos en keikis por las aplicaciones de Round Up, agrupadas junto con la curvatura de las hojas y la producción de plántulas, lo cual indica que dichos efectos son producidos por las aplicaciones de glifosato (Fig. 7). Se puede observar que en la dosis 0.1 hubo un considerable número de keikis al igual que en la dosis 1 equivalente a la concentración 544.5 g/l (Fig. 6). 


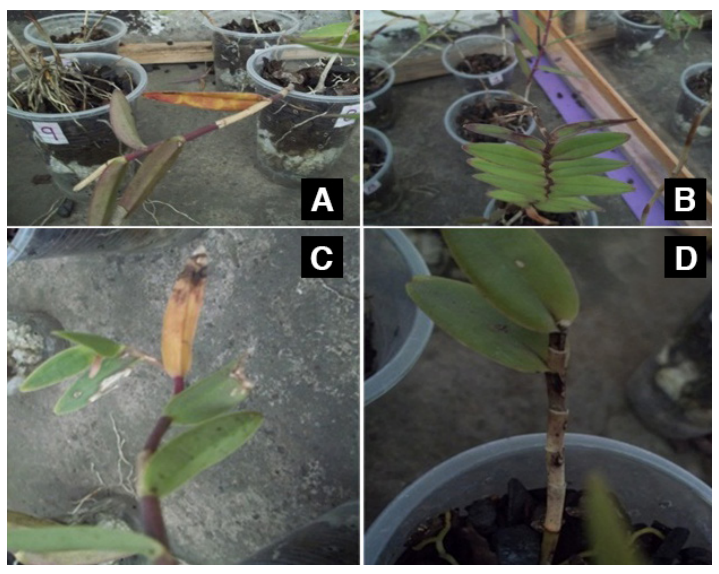

Figura 5. A. Hojas mareadas y amarillas. B. Hojas con puntos morados y curveadas. C. Hojas amarillas con café, con puntos negros y blancos. D. Tallo negro.

Número de hojas: se presentaron mayores pérdidas de hojas en las dosis 1 y 0.1 con respecto al control, al igual se presentan diferencias significativas entre las aplicaciones previas y posteriores a la aplicación del Round Up, pues $\operatorname{Pr}>\mathrm{F}=0.0002<$ 0.05 (Fig. 8).

Los cortes histológicos a nivel microscópico de las afectaciones en hoja y tallo de E. melinanthum (Fig. 9), indicaron que en el tallo se afecta el haz vascular, dentro del cual, el xilema y el floema se ven deformados y quemados por el químico (Fig. 9D), por su parte, las hojas se encuentran afectadas principalmente la cutícula y la epidermis, pues presentan deformidades y pudrimiento en algunos sectores de las células (Fig. 9F, Fig. 9G y Fig. 9H).

\section{Discusión}

Los hongos aislados de las raíces de $E$. melinanthum se ven afectados por el glifosato con dosis aplicadas en la caña de azúcar. El efecto mayor se ve en la concentración de $544.5 \mathrm{~g} / 1$ pero se evidencia que también tiene efecto en la dosis $1 / 10$ con respecto al control. Este tipo de patrón se observó también en la perdida de hojas, pero a dichas concentraciones, el glifosato puede llegar a generar una considerable cantidad de keikis como un medio de respuesta para la supervivencia de la orquídea. Zhao et al. en 2014 detectaron el éxito que tiene el glifosato a la hora de dañar el cultivo pues las hojas recién nacidas que

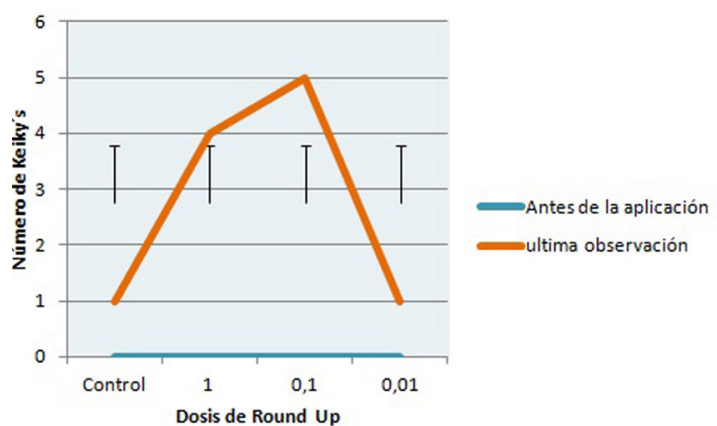

Figura 6. Producción de keikis antes y después de la aplicación de glifosato en las dosis estudiadas. Las barras representan el error con desviación estándar con tamaño de muestra de 30 plántulas por cada dosis.

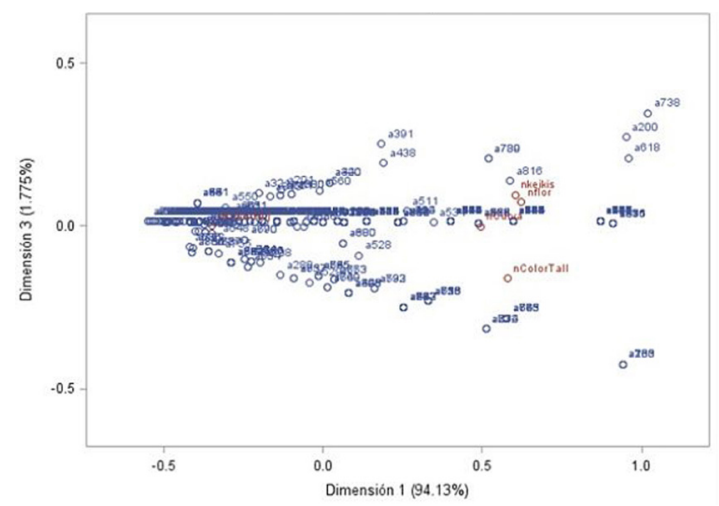

Figura 7. Semejanzas entre los efectos presentados en keikis, color de tallo, color de hojas y curvatura.

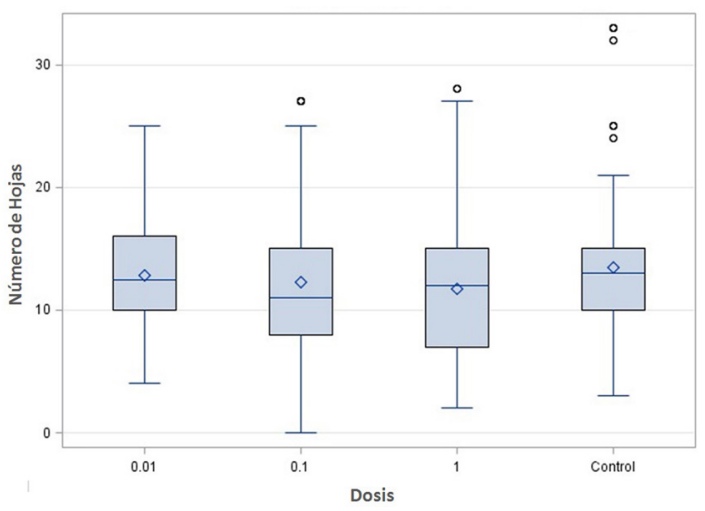

Figura 8. Pérdida de hojas por tratamiento durante el tiempo del estudio. La línea del centro de la caja representa la mediana, el diamante representa la media y los bigotes representan los percentiles 5\% - 25\% y $75 \%$ - $95 \%$ y la caja representa el $50 \%$ equivalente a la resta del cuartil tres y cuartil uno. Los puntos que se encuentran por fuera de los bigotes son datos atípicos 


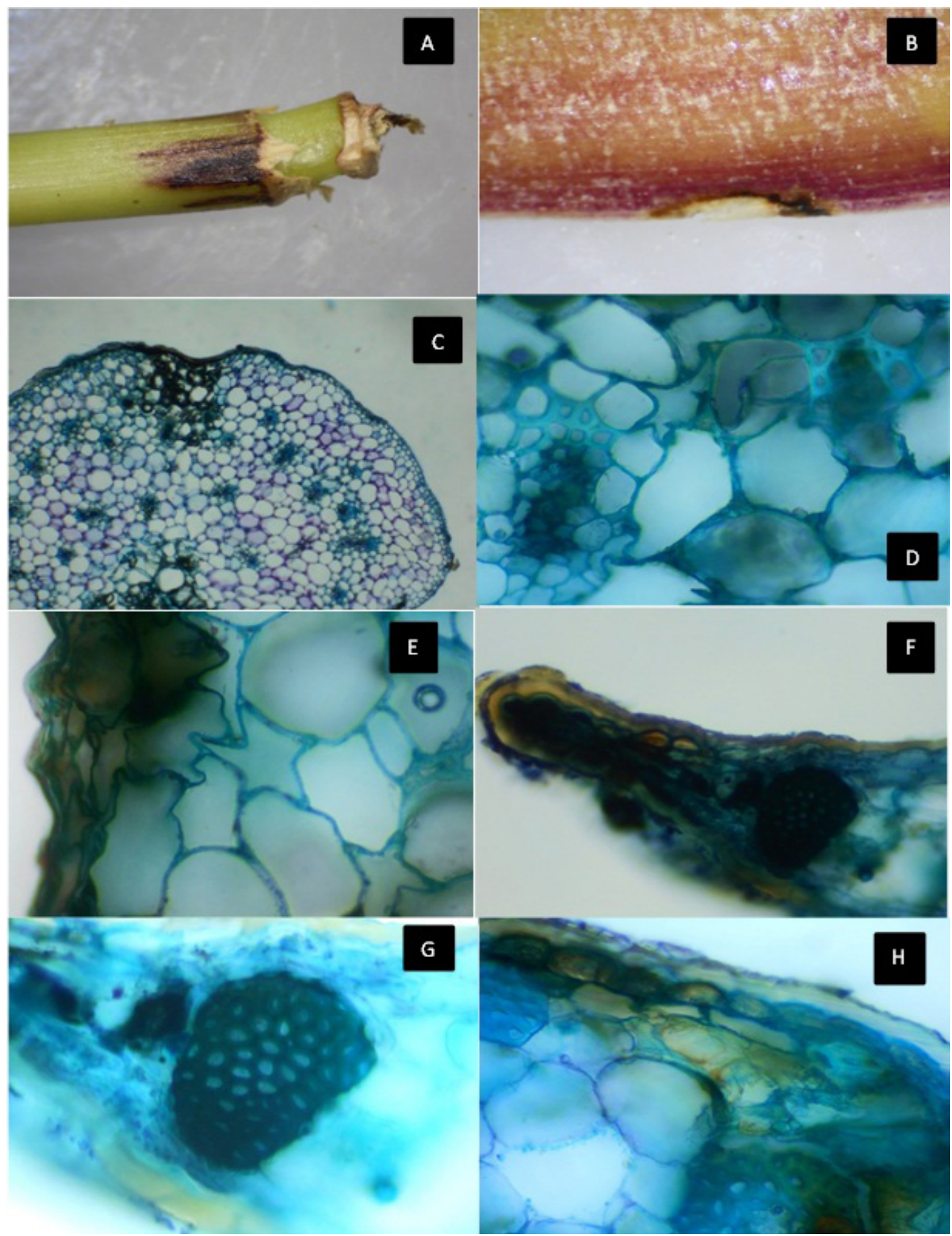

Figura 9. A. Segmento de tallo afectado. B. Segmento de hoja afectada. C. Efecto de glifosato en tallo a 4X. D. Afectación en haz vascular a 40X. E. Afectación en el velamen a 40X. F. Corte transversal de hoja donde se observa la quemazón y afectación en esclerénquima a 10X. G. Daño en Esclerénquima a 40X. H. Daño en cutícula y epidermis. 
generalmente se encuentran en la zona superior de las plantas son más sensibles a la disminución de la clorofila, por otra parte obtuvo que la bioquímica foliar es un indicador potencialmente para la detección temprana del estrés que presentan las plantas a causa de las lesiones del glifosato, pues las lesiones se pueden detectar pasadas 48 horas de la aplicación.

Si bien el glifosato inhibe el crecimiento de unos hongos aislados de E. melinanthum, también puede reducir la viabilidad, la germinación y el crecimiento de las esporas de los hongos, así como la colonización de hongos micorrízicos, pues los hongos pueden verse afectados en zonas aledañas a la aplicación del herbicida e incluso cuando se ve impulsado por la deriva del viento (Druille et al. 2013). Otro tipo de afectación que puede ser aún más grave es que el glifosato puede llegar vía indirecta a los hongos, es decir, que el herbicida pude cambiar los suministros de hidratos de carbono (Druille et al. 2013), pues el glifosato puede llegar a reducir la fuente energética de la planta o a la destrucción de la pared celular de la orquídea conduciendo de esta manera a la muerte de la planta.

Dosis altas de glifosato afectan principalmente las hojas de E. melinanthum que se evidencian en el cambio de coloración, la caída de las hojas, la inhibición de la fotosíntesis, lo que puede conllevar a la muerte la planta. Dado que, el glifosato impacta la tasa fotosintética pues reduce el contenido de clorofila en las hojas cuando se aplica en el follaje de las plantas (Druille et al. 2013), pues los fotorreceptores situados en la clorofila son los encargados de absorber de manera eficaz la luz azulvioleta $(450-500 \mathrm{~nm})$ y rojo-naranja $(650-750 \mathrm{~nm})$, pues la fotosíntesis es más eficiente para las plantas en la longitud de onda citada (Gliessman 2002). Dado lo anterior, se puede observar las diversas sintomatologías causadas por el glifosato en las hojas produciendo una marchitez que termina en la perdida de las hojas o privando la respiración a la planta.

El Round Up aplicado no actúa sistémicamente en las orquídeas, pues lo que genera son afectaciones localizadas, por lo que se considera un agroquímico contacto cuando actúa a dosis altas en E. melinanthum, afectando negativamente el velamen en el tallo, la cutícula, el haz vascular y la epidermis en hojas, lo cual puede ocasionar desecación de las hojas y desprotección de la orquídea a los ataques de bacterias y hongos (Boom et al. 2005). Se puede deducir que se presenta curvatura en las hojas de la orquídea porque detiene el crecimiento de algunas células, lo que se visualiza puntualmente en la distorsión de la forma normal de la hoja.

\section{LITERATURA CITADA}

ASOCAÑA, (2012). El sector azucarero en la actualidad. http://www.asocana.org/publico/info.aspx?Cid=215; Consulta: febrero 2015.

Beyrle, H. F., Smith, S. E., Franco, C. M. M., \& Peterson, R. L. (1995). Colonization of Orchis morio protocorms by a mycorrhizal fungus: effects of nitrogen nutrition and glyphosate in modifying the responses. Canadian Journal of Botany, 73(8), 1128-1140. DOI: 10.1139/b95-123

Boom, A., Sinninge, J. S., De Leeuw, J. W. (2005). Cutan, a common aliphatic biopolymer in cuticles of drought-adapted plants. Organic Geochemistry, 36 (4), 595-601. DOI: 10.1016/j.orggeochem.2004.10.017

Cassalett, D. C., Torres, A. J., Isaacs, E. C. (1995). El cultivo de la caña en la zona azucarera de Colombia. 1th ed. Cali, Cenicaña, $394 \mathrm{p}$.

Cox, C. (1998). Glyphosate (Round up). Journal of Pesticide Reform, 18(3), 3-15.

Druille, M., Omacini, M., Golluscio, R. A., Cabello, M. N. (2013). Arbuscular mycorrhizal fungi are directly and indirectly affected by glyphosate application. Applied Soil Ecology, 72, 143-149. DOI: 10.1016/j.apsoil.2013.06.011

FAO. (2016). Herbicide action. (Consultado: 1 de julio de 2016, http://www.fao.org/ag/agp/agpc/doc/Publicat/FAOBUL2/ B206.htm).

Freitas, M. R., Barigye, S. J., Daré, J. K., Freitas, M. (2015). Aug-MIA-SPR/PLS-DA classification of carbonyl herbicides according to levels of soil sorption. Geoderma, 268, 1-6. DOI: 10.1016/j.geoderma.2016.01.013

Gliessman, S. R. (2002). Introducción. En: Agroecología. En: Procesos ecológicos en agricultura sostenible (pp. 385). Costa Rica: LITOCAT.

Han, J. Y., Xiao, H., Jiangyun, G. (2016). Seasonal dynamics of mycorrhizal fungi in Paphiopedilum spicerianum (Rchb. f) Pfitzer - A critically endangered orchid from China. Global Ecology and Conservation, 6, 327-338. DOI: 10.1016/j. gecco.2016.03.011 
Hajebi, A., Das, T. K., Arora, A., Bala, S., Hajebi, F. (2015). Herbicides tank-mixes effects on weeds and productivity and profitability of chilli (Capsicum annuum L.) under conventional and zero tillage. Scientia Horticulturae, 198, 191-196. DOI: $10.1016 /$ j.scienta.2015.11.020

Landra, R., Caseley, J. C., Parker, C. (1996). Herbicidas. Pp.193-195 en: Manejo de Malezas para Países en Desarrollo (Estudio FAO Producción y Protección Vegetal - 120). Roma: FAO.

Mosquera-Espinosa, A.T., Bayman, P., Otero J. T. (2010). Ceratobasidium como hongo micorrízico de orquídeas en Colombia. Acta Agronómica, 59(3), 316-326.

Obour, A. K., Stahlman, P. W., Holman, J. D. (2016). Soil chemical properties as influenced by long-term glyphosateresistant corn and soybean production in the central Great Plains, USA. Geoderma, 277, 1-9. DOI: 10.1016/j. geoderma.2016.04.029.

Otero J.T, Ackerman J.D, Bayman P. (2002). Diversity and Host Specificity of Endophytic Rhizoctonia-like Fungi from Tropical Orchids. American Journal of Botany, 89(11), 1852-1858.

Otero, J. T., Ackerman, J. D., Bayman, P. (2004) Differences in mycorrhizal preferences between two tropical orchids. Molecular Ecology, 13(8), 2393-2404. DOI: 10.1111/j.1365-294X.2004.02223.x

Otero, J. P., Bayman, P., Ackerman, J. D. (2005). Variation in mycorrhizal performance in the epiphytic orchid Tolumnia variegata in vitro: the potential for natural selection. Evolutionary Ecology, 19(1), 29-43. DOI: 10.1007/s10682-0045441-0

Otero, J. P., Flanagan, N. S., Herre, E. A., Ackerman, J. D., Bayman, P. (2007). Widespread mycorrhizal specificity correlates to mycorrhizal function in the neotropical, epiphytic orchid Ionopsis utricularioides (Orchidaceae). American Journal of Botany, 94(12), 1944-1950. DOI: 10.3732/ajb.94.12.1944

Otero, J.T., Bayman, P. (2009). Germinación simbiótica y asimbiótica en semillas de orquídeas epifitas. Acta Agronómica, 58(2), 270-276.

Panwar, D., Ram, K., Harish, Shekhawat N. S. (2012). In vitro propagation of Eulophia nuda Lindl., an endangered orchid. Scientia Horticulture, 139, 46-52. DOI: 10.1016/j.scienta.2012.01.011

Pereira, G., Romero, C., Suz, L. M., Atala, C. (2014). Essential mycorrhizal partners of the endemic Chilean orchids Chloraea collicensis and C. gavilu. Flora - Morphology, Distribution, Functional Ecology of Plants, 209,. 95-99. DOI: 10.1016/j.flora.2013.12.001

Pillon, Y., Fay, M. F., Shipunov, A. B., Chase, M. W. (2006). Species diversity versus phylogenetic diversity: A practical study in the taxonomically difficult genus Dactylorhiza (Orchidaceae). Biological Conservation, 129, 4-13. DOI: 10.1016/j.biocon.2005.06.036

Puerto, K., Quiroz, A., Talavera, C., Santamaría, J. (2001). Análisis Histológico de Hojas de Plantas de Cocos nucifera L. Cultivadas in vitro. En A. Aguilar, IX Congreso Nacional de Biotecnología y Bioingeniería, XIII Congreso Nacional de Ingeniería Bioquímica y II Congreso Internacional de Ingeniería Bioquímica. Sociedad Mexicana de Biotecnología y Bioingeniería, Veracruz-México.

Rankin, B. L., Ballantyne, M., Pickering, C. M. (2015). Tourism and recreation listed as a threat for a wide diversity of vascular plants: A continental scale review. Journal of Environmental Management, 154, 293-298. DOI: 10.1016/j. jenvman.2014.10.035

Reina-Rodríguez, G. A. \& Otero, J. T. (2011). Guía ilustrada de las orquídeas del Valle Geográfico del río Cauca y Piedemonte Andino.

Reina-Rodríguez, G. A., Ospina-Calderón, N. H., Castaño, A., Soriano, I., Otero, J. T. (2010). Catálogo de las orquídeas del Valle Geográfico del río Cauca y su piedemonte Andino Bajo. Sur - occidente colombiano. CESOEDESIA, 32(90), 91.

Romero, G. A. Ferreira, I. (2006). Index of botanical specimens. En: Harvard University herbaria \& libraries, http://kiki. huh.harvard.edu/databases/specimen_search.php?mode=details\&id[]=155268; consulta: noviembre 2015.

Steinmann, H. H., Dickeduisberg, M., Theuvsen, L. (2012). Uses and benefits of glyphosate in German arable farming. Crop Protection, 42, 164-169. DOI: 10.1016/j.cropro.2012.06.015

$\mathrm{Su}$, Y. L., Dela Cruz, A., Moore, P. H., Maretzki, A. (1992). The Relationship of Glyphosate Treatment to Sugar Metabolism in Sugarcane: New Physiological Insights. Journal of Plant Physiology, 140, 168-173. DOI: 10.1016/S01761617(11)80929-6

Tan, M. X., Wang, C. L., Chen X. M.., Zhou, Y.Q., Wang, Y. Q., Luo, A. U., Liu, Z. H., Guo, S. X. (2014). In vitro seed germination and seedling growth of an endangered epiphytic orchid, Dendrobium officinale, endemic to China using mycorrhizal fungi (Tulasnella sp.). Scientia Horticulturae, 165, 62-68. DOI: 10.1016/j.scienta.2013.10.031

Valadares, R. B. D. S., Otero, J. T., Pereira, M. C., \& Cardoso, E. J. B. N. (2015). The epiphytic orchids Ionopsis 
utricularioides and Psygmorchis pusilla associate with different Ceratobasidium lineages at Valle del Cauca, Colombia. Acta Botanica Brasilica, 29(1), 40-44.

Zhang, Y., Lee, Y., Deng, L., Zhao, S. (2013). Asymbiotic germination of immature seeds and the seedling development of Cypripedium macranthos Sw., an endangered lady's slipper orchid. Scientia Horticulturae, 164, 130-136. DOI: 10.1016/j.scienta.2013.08.006

Zhang, Z., Yan, Y., Tian, Y., Li, J., He, J., Tang, Z. (2015). Distribution and conservation of orchid species richness in China. Biological Conservation, 181, 64-72. DOI: 10.1016/j.biocon.2014.10.026.

Zhao, F., Guo, Y., Huang, Y., Reddy, K., Lee, M. A., Fletcher, R. S., Thomson, S. J. (2014). Early detection of crop injury from herbicide glyphosate by leaf biochemical parameter inversion. International Journal of Applied Earth Observation and Geoinformation, 31, 78-85. DOI: 10.1016/j.jag.2014.03.010 